\title{
Prevalence and Antimicrobial Susceptibility pattern of Methicillin Resistant Staphylococcus aureus Isolates at a tertiary Care Hospital in Rajkot, Western India
}

\author{
Nirali Daftary*" and Krunal Mehta \\ Department of Microbiology, P.D.U Medical College \& Hospital, Rajkot-360001, \\ Gujarat, Western India \\ *Corresponding author
}

\begin{abstract}
A B S T R A C T
Prevalence and Antimicrobial Susceptibility Pattern of Methicillin Resistant Staphylococcus aureus Isolates at a tertiary Care Hospital in Rajkot, Western India. Aim of the study is to analyse the prevalence and the antimicrobial Susceptibility pattern of

Keywords

MRSA, Prevalence

Article Info

Accepted:

04 April 2018

Available Online:

10 May 2018

Methicillin Resistant Staphylococcus aureus (MRSA). A total of 300 clinical samples were taken from various sites and the pathogen was identified as Staphylococcus aureus based on gram stain, various biochemical tests and culture. Subsequently the antibiotic susceptibility test was performed and MRSA was detected from it. Out of the total 300 samples of S. aureus, 76 (25\%) were found to be Methicillin Resistant Staphylococcus aureus (MRSA). Hospital acquired MRSA was more common than Community Acquired MRSA. All clinical samples were resistant to penicillin, $48.68 \%$ to ciprofloxacin and levo floxacin, $23 \%$ to cotrimoxazole, $9.21 \%$ to tetracycline, $6.57 \%$ to gentamycin, $3.94 \%$ to chloramphenicol. All the samples were sensitive to linezolid, vancomycin and rifampicin. Conclusion: The regular surveillance of hospital-acquired infections of MRSA may be helpful in formulating and monitoring the antibiotic policy \& in preserving antibiotics like vancomycin, only for life-threatening staphylococcal diseases.
\end{abstract}

\section{Introduction}

Methicillin Resistant Staphylococcus is a leading cause of gram positive bacterial infections and produces a wide spectrum of diseases, ranging from minor skin infections to fatal systemic ones (Debbie Josefson, 1997). After the emergence of MRSA as a nosocomial pathogen in the early 1960s, there has been an increase in the number of MRSA outbreaks both in the hospitals and the community at large (Bruce R. Lyon et al., 1987). Early detection of MRSA and formulation of effective antibiotic policy in tertiary care hospitals is of paramount importance from the epidemiological point.

The present study has been carried out in our teaching hospital with an aim to know the prevalence and antibiotic susceptibility pattern of Methicillin Resistant Staphylococcus aureus isolates, in order to utilize the 
information obtained and formulate the antibiotic policy and appropriate control measures.

\section{Materials and Methods}

This study was aimed to find the prevalence of MRSA and its susceptibility pattern at our teaching hospital at Rajkot, West India, for a period of 9 months. A total of 300 isolates of $S$. aureus were obtained from various clinical specimens of different wards and OPDs like pus, wound swab, blood, sputum, urine, body fluids, vomitus and food.

The specimens were cultured on blood agar and MacConkey agar plates and incubated aerobically at $37^{\circ} \mathrm{C}$ for 48 hours (Gerald Collee et al., 1996). The isolates were identified using standard tests like gram stain, catalase, slide and tube coagulase and mannitol test (Washington et al., 2005).

They were then subjected to antibiotic susceptibility testing by modified Kirby Bauer's disc diffusion method on Muller Hinton Agar plates using various antibiotic discs like penicillin $\mathrm{G}$ (10 units).

Gentamicin (10ug), levofloxacin (5ug), ciprofloxacin (5ug), erythromycin (15ug), clindamycin (2ug), tetracycline (30ug), cotrimoxazole $\quad(1.25 / 23.75 \mathrm{ug}), \quad$ linezolid (30ug), chloramphenicol (30ug) as per CLSI guidelines.

Test for methicillin resistance was performed by Kirby-Bauer disc diffusion method using cefoxitin (30ug) disc on Muller-Hinton agar (HiMedia Labs, Mumbai). The agar plate was previously inoculated with 0.5 McFarland standard bacterial suspension, the cefoxitin disc (30ug) was placed \& after 16-18 hours of incubation at $33-35^{\circ} \mathrm{C}$, the zone of inhibition was observed (Clinical Laboratory Standards Institute, 2017).
All S.aureus strains were tested for susceptibility to cefoxitin disc diffusion test for detection of MRSA (Clinical Laboratory Standards Institute, 2017) along with other antibiotic discs.

MRSA - $21 \mathrm{~mm}$ or less diameter of inhibition zone of cefoxitin

MSSA - $21 \mathrm{~mm}$ or more diameter of inhibition zone of cefoxitin

Cefoxitin is used as a surrogate for mecAmediated oxacillin resistance (Clinical Laboratory Standards Institute, 2017). Isolates that test as mecA positive should be reported as oxacillin (not cefoxitin) resistant; routine testing of other $\beta$-lactam agents, except those with anti-MRSA activity, is not advised.

Quality control (QC) of the various tests and also of the antibiotic discs was performed with S. aureus ATCC 25923, according to the standard disc diffusion QC procedure. Additional QC was performed with separate in-house selected $S$. aureus strains that demonstrated sensitive and resistant to cefoxitin.

\section{Results and Discussion}

A total of 300 isolates of Staphylococcus aureus were studied over a period of 9 months in the Microbiology Laboratory at a tertiary hospital, Rajkot. Of them 25\% (76) samples were found to be of MRSA and 75\% (224) samples were found to be of MSSA.

Out of all the 76 samples, 69.7\% (53) samples were of pus (SSTI), blood samples were $25 \%$ (19), DLC tip, pleural fluid, sputum and urine samples were $1.31 \%$ (1) each. Females were predominant with $54 \%$ (41) than males at $46 \%$ (35). The age group distribution is as follows; 41-50 years were maximum at $23.60 \%$, followed by 31-40years at $17.10 \%, 21-30$ 
years and 51 -60 years were at $15.70 \%, 01-10$ years were at $10.50 \%, 11-20$ years at $9.21 \%$, $61-70$ years at $5.26 \%$, and $71-80$ at $2.63 \%$ (Table 1). Based on the patients' history and admission, 34\% (26 isolates) had CA-MRSA and 66\% (50 isolates) had HA-MRSA.

The antibiotic resistance in MRSA was as follows: Penicillin was maximum with $100 \%$ (76), Ciprofloxacin 48.68\% (37), Levofloxacin $48.68 \%$ (37), cotrimoxazole $23 \%$ (18), Tetracycline $9.21 \%$ (7), Gentamicin $6.57 \%$ (5), Chloramphenicol 3.94\% (3) and no resistance to vancomycin, linezolid and rifampicin. In urinary samples, resistance was seen in trimethoprim while no resistance was seen in norfloxacin and nitrofurantoin (Table 2 ). In the present study, of the total 300 samples, MRSA is seen in 25\% (76) of the isolates and MSSA in 75\% (224) of the isolates. Similar results were observed by Gupta et al., (2009), Jadhav Savita Vivek et al., (2011), INSAR group (2013), McNeil et al., (2013) and Yoo Sang et al., (2016) (Table 3). On the contrary, some studies have reported alarming high incidence of MRSA; reports from a Delhi hospital have shown the MRSA prevalence of 51.6\% in 2001 (Tiwari et al., 2008), whereas it was reported as $38.44 \%$ in the same hospital in 2008. The epidemiology of MRSA over different parts of India is not uniform.

Table.1 Age wise distribution

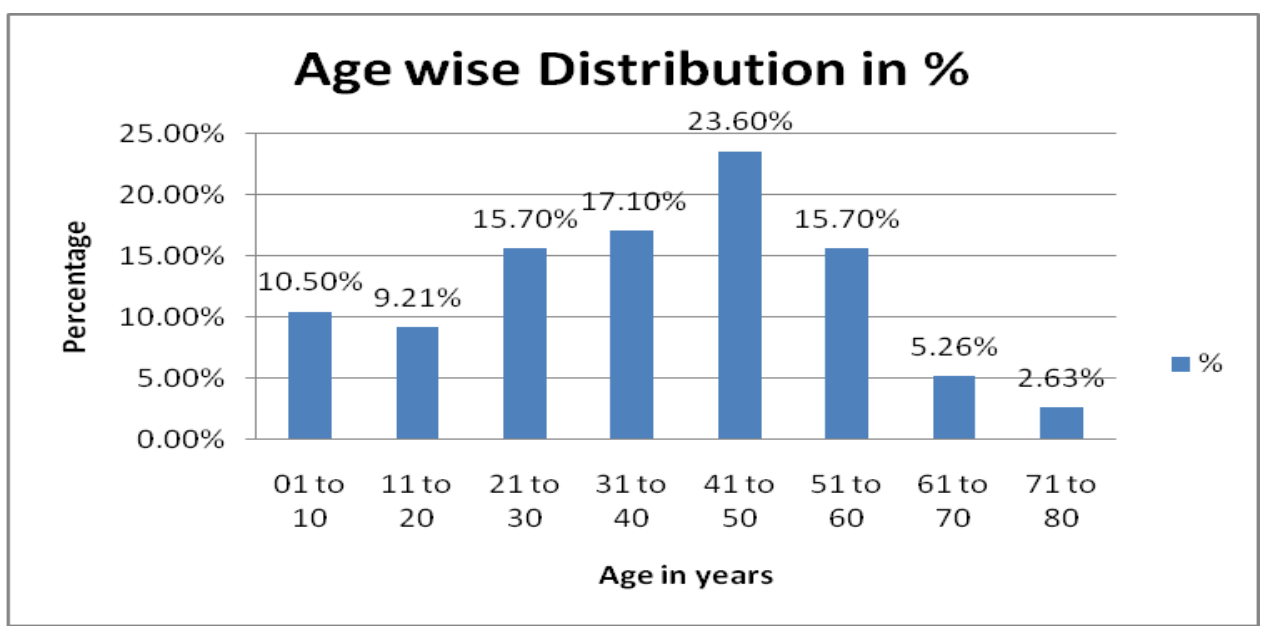

Table.2 Antimicrobial resistance in MRSA samples

\begin{tabular}{|c|c|c|}
\hline Drugs & $\mathbf{R}$ & $\mathbf{S}$ \\
\hline Cotrimoxazole & $18(23 \%)$ & 58 \\
\hline Tetracycline & $7(9.21 \%)$ & 69 \\
\hline Chloramphenicol & $3(3.94 \%)$ & 73 \\
\hline Ciprofloxacin & $37(48.68 \%)$ & 39 \\
\hline Levofloxacin & $37(48.68 \%)$ & 39 \\
\hline Gentamicin & $5(6.57 \%)$ & 71 \\
\hline Penicillin & $76(100 \%)$ & 0 \\
\hline Vancomycin & 0 & 76 \\
\hline Linezolid & 0 & 76 \\
\hline Rifampicin & 0 & 76 \\
\hline
\end{tabular}


Table.3 Methicillin sensitivity

\begin{tabular}{|c|c|c|c|}
\hline STUDY & YEAR & MRSA & MSSA \\
\hline V Gupta et al., & 2009 & $25 \%$ & $75 \%$ \\
\hline Jadhav et al., & 2011 & $32.5 \%$ & $67.84 \%$ \\
\hline INSAR group & 2013 & $41 \%$ & $59 \%$ \\
\hline McNeil et al., & 2013 & $31 \%$ & $69 \%$ \\
\hline Yoo Sang et al., & 2016 & $47.4 \%$ & $52.6 \%$ \\
\hline Present Study & 2016 & $25.33 \%$ & $74.67 \%$ \\
\hline
\end{tabular}

Table.4 Hospital acquired and community acquired MRSA

\begin{tabular}{|c|c|c|c|}
\hline STUDY & YEAR & HA -MRSA & CA - MRSA \\
\hline Debasmita et al., & 2013 & $65 \%$ & $35 \%$ \\
\hline Kiran et al., & 2015 & $54.85 \%$ & $45.15 \%$ \\
\hline Abbas A et al., & 2015 & $71.1 \%$ & $28.9 \%$ \\
\hline Present Study & 2016 & $66 \%$ & $34 \%$ \\
\hline
\end{tabular}

Table.5 Based on site

\begin{tabular}{|c|c|c|c|}
\hline STUDY & YEAR & SSTI & OTHER SITES \\
\hline Timothy et al., & 2003 & $75 \%$ & $25 \%$ \\
\hline Scott et al. & 2005 & $77 \%$ & $23 \%$ \\
\hline Present Study & 2016 & $67 \%$ & $33 \%$ \\
\hline
\end{tabular}

Multidrug resistance among MRSA strains was higher than those that were susceptible to methicillin. Ciprofloxacin was proposed to be an alternate therapy for MRSA infection (Sharon et al., 1989). Although rapidly developing resistance to ciprofloxacin has been reported, the antibiotic was found working on $S$. aureus in our hospital. This is perhaps due to the differential clonal expansion and drug pressure in the community. In the present study, based on the patients' history and admission, $66 \%$ MRSA was found to be Hospital acquired and $34 \%$ MRSA was found to be community acquired. Similar results were observed by Debasmita et al., (2013), Kiran et al., (2015) and Abbas et al., (2015) (Table 4).

In the present study, out of 300 cases, $67 \%$ (201) samples were of skin and soft tissue infections. 33\% (99) cases were from other sites including systemic infections and blood cultures. Similar observations were made by
Timothy et al., (2003) and Scott et al., (2005) (Table 5).

\section{Acknowledgements}

Authors thank the entire staff of the P.D.U Medical College and civil hospital, Rajkot for providing support for recruitment of patients and collection of samples. We also thank the Department of Microbiology, Rajkot for extending support for the study.

\section{References}

Abbas, A, Nirwan, P.S., Srivastava, P. Prevalence and antibiogram of hospital acquired-methicillin resistant Staphylococcus aureus and community acquired-methicillin resistant Staphylococcus aureus at a tertiary care hospital National Institute of Medical Sciences. Community Acquir Infect 2015; 2: $13-5$. 
Bruce R. Lyon and Ron Skurray, et al., Antimicrobial resistance of Staphylococcus aureus, Genetic basis: Microbiological Reviews: March 1987: Volume-51(1), p. 88 - 134.

Clinical Laboratory Standards Institute, 2017.

Debasmita Dubey, Shakti Rath, et al., A report on infection dynamics of inducible clindamycin resistance of Staphylococcus aureus isolated from a teaching hospital in India. doi:10.1016/S22211691(13)60040-4.

Debbie Josefson, 1997: Vancomycin resistant Staphylococcus aureus reported: British Medical Journal: 20 September: Volume315 , p. 700

Gerald Collee, J., Barrie P, et al., 1996. Mackie and McCartney Practical Medical Microbiology. 4th edn.

Gupta V, Datta P, Rani H, Chander J. Inducible clindamycin resistance in Staphylococcus aureus: A study from North India. J Postgrad Med 2009; 55: 176-9.

Indian Network for Surveillance of Antimicrobial Resistance (INSAR) group, India. Methicillin resistant Staphylococcus aureus (MRSA) in India: prevalence \& susceptibility pattern. Indian J Med Res. 2013 Feb; 137(2): 363 9.

Jadhav Savita Vivek, Gandham Nageswari Rajesh, et al., Prevalence of inducible Clindamycin resistance among community-and hospital-associated Staphylococcus aureus isolates in a tertiary care hospital in India. Biomedical Research 2011; 22 (4): 465-469.

Kiran K Mokta, Santwana Verma, et al., Inducible Clindamycin Resistance among Clinical Isolates of Staphylococcus aureus from Sub Himalayan Region of
India. Int J Adv Med. 2015; 2(3): 264268.

McNeil, J. Chase, et al., Staphylococcus aureus Infections in Pediatric Oncology Patients: High Rates of Antimicrobial Resistance, Antiseptic Tolerance and Complications. Pediatric Infectious Disease Journal: February 2013: Volume 32: Issue 2: p. 124-128.

Scott K. Fridkin, Jeffrey C. Hageman, et al., Methicillin-Resistant Staphylococcus aureus Disease in Three Communities. N Engl J Med 2005; 352:1436-1444 April 7, 2005DOI: 10.1056/NEJMoa 043252

Sharon MS, Robert HK, Flor TT. Ciprofloxacin in therapy for Methicillin Resistant Staphylococcus aureus infections or colonizations. Antimicrob Agents Chemother. 1989; 2: 181-4.

Timothy S. Naimi, Kathleen H. LeDell, et al., Comparison of Community- and Health Care-Associated Methicillin-Resistant Staphylococcus aureus Infection. JAMA. 2003; 290(22): 2976-2984. doi:10.1001/jama.290.22.2976.

Tiwari HK, Sapkota D, Sen MR. High prevalence of multidrug-resistant MRSA in a tertiary care hospital of northern India. Infection and Drug Resistance 2008; 1: 57-61.

Washington W, Stephen A, et al., 2005. Koneman's Colour Atlas and Textbook of Diagnostic Microbiology. 6th edn. Philadelphia; Lippincott Williams and Wilkins.

Yoo Sang, Jiehyun Jeon, et al., Antimicrobial resistance of Staphylococcus aureus isolated from skin infections and its implications in various clinical conditions in Korea. 19 February 2016.

\section{How to cite this article:}

Nirali Daftary and Krunal Mehta. 2018. Prevalence and Antimicrobial Susceptibility pattern of Methicillin Resistant Staphylococcus aureus Isolates at a tertiary Care Hospital in Rajkot, Western India. Int.J.Curr.Microbiol.App.Sci. 7(05): 373-377. doi: https://doi.org/10.20546/ijcmas.2018.705.048 